\title{
Assessment of swimming associated health effects in marine bathing beach: An example from Morib beach (Malaysia)
}

\begin{abstract}
A survey among beachgoers was conducted to determine the swimming associated health effects experienced and its relationship with beach water exposure behaviour in Morib beach. For beach water exposure behaviour, the highest frequency of visit among the respondents was once a year $(41.9 \%)$. For ways of water exposure, whole body exposure including head was the highest (38.5\%). For duration of water exposure, $30.8 \%$ respondents prefer to be in water for about $30 \mathrm{~min}$ with low possibilities of accidental ingestion of beach water. A total of $30.8 \%$ of beachgoers in Morib beach were reported of having dermal symptoms. Bivariate analysis showed only water activity, water contact and accidental ingestion of beach water showed significant association with swimming associated health effects experienced by swimmers. This study output showed that epidemiological study can be used to identify swimming associated health effects in beach water exposed to faecal contamination.
\end{abstract}

Keyword: $\quad$ Beach; Recreational activities; Water exposure; Health effects 